we set out to guide lead placement by avoiding scar and targeting the region of the LV with the latest mechanical activation.

Methods 17 patients underwent cardiac magnetic resonance (CMR) scans. 3D whole heart images were segmented to produce high fidelity anatomical models of the cardiac chambers and coronary veins. 2, 3, 4 chamber and short axis cine images were processed using Tomtec software to give a 16 segment time volume-dyssynchrony map. In patients with myocardial scar the late gadolinium enhancement images were manually segmented and registered to the anatomical model along with the dyssynchrony map. The 3 latest mechanically activated segments with $<50 \%$ scar were identified and this information was overlaid at CRT implant on to live fluoroscopic images using a prototype version of the Philips EP Navigator software. Subsequently, the x-ray C-arm and table could be moved freely while automatically maintaining a registered roadmap. We used a high fidelity pressure wire to assess the acute haemodynamic response to pacing in different regions of the overlaid 16 segment model. All $\mathrm{dP} / \mathrm{dt}$ measurements were compared to baseline AAI or VVI (for those patients in AF) pacing at $5-10$ beats/min above intrinsic rate.

Results 15 of the 17 patients underwent successful placement of a LV pacing lead via the CS with satisfactory pacing parameters and no phrenic nerve stimulation at implant. The mean time from insertion of the CS guide catheter into the venous sheath to successful cannulation of the CS was $1.3 \pm 1.0 \mathrm{~min}$. In 2 patients we were unable to place a LV lead successfully in any branch of the CS. We paced in at least one of our 3 target segments in 11 patients. $67 \%$ of patients were responders as defined by a $10 \%$ increase in $+\mathrm{dP} / \mathrm{dt}$ over baseline. The mean change in $+\mathrm{dP} / \mathrm{dt}$ for the best lead position vs baseline $+\mathrm{dP} / \mathrm{dt}$ was $15.9 \pm 11.3 \%$ for DDDLV pacing. This compares to a mean change in $+\mathrm{dP} / \mathrm{dt}$ of $14.9 \pm 12.3 \%$ when the CMR dyssynchrony-map defined target region was paced DDDLV. The region of best $+\mathrm{dP} / \mathrm{dt}$ response was postero-lateral, lateral or posterior in all cases.

Conclusion We have shown it is feasible to acquire, overlay and accurately register cardiac MR data on to fluoroscopic images at the time of CRT implant. Our data suggest that it is also possible to identify and place the LV lead in at least one target region in most patients. This appears to give close to the best acute haemodynamic response that can be achieved in any branch of the CS. The initial results of this pilot study suggest that a MR dyssynchrony guided approach to LV lead placement may allow ideal LV lead positioning (Abstract 152 figures 1 and 2).

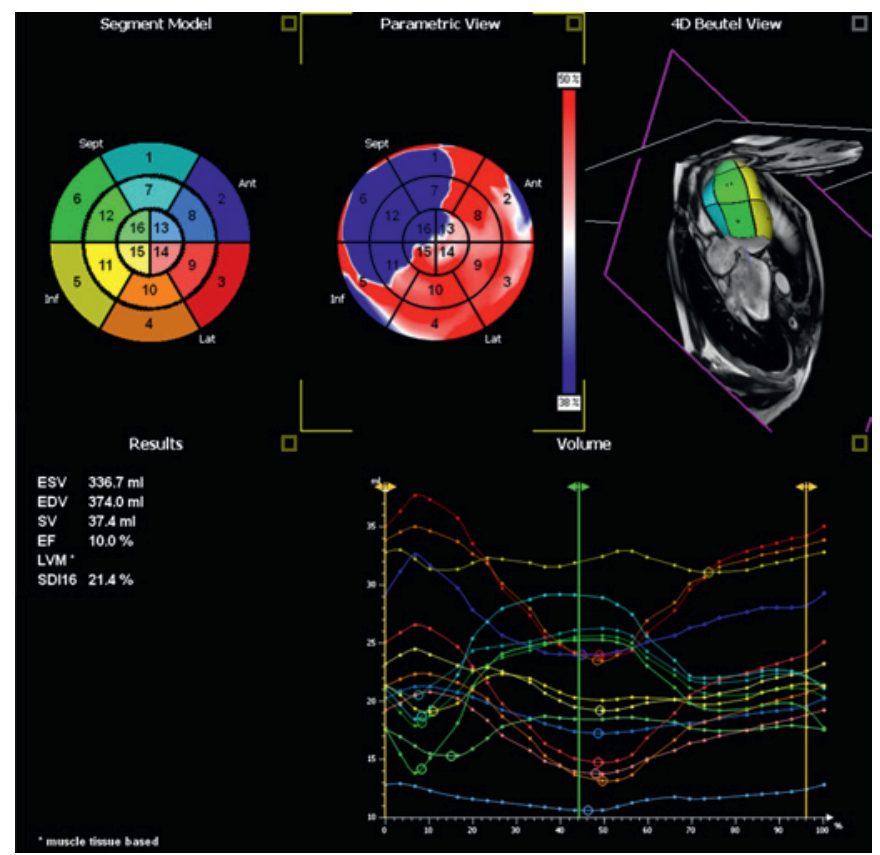

Abstract 152 Figure 1

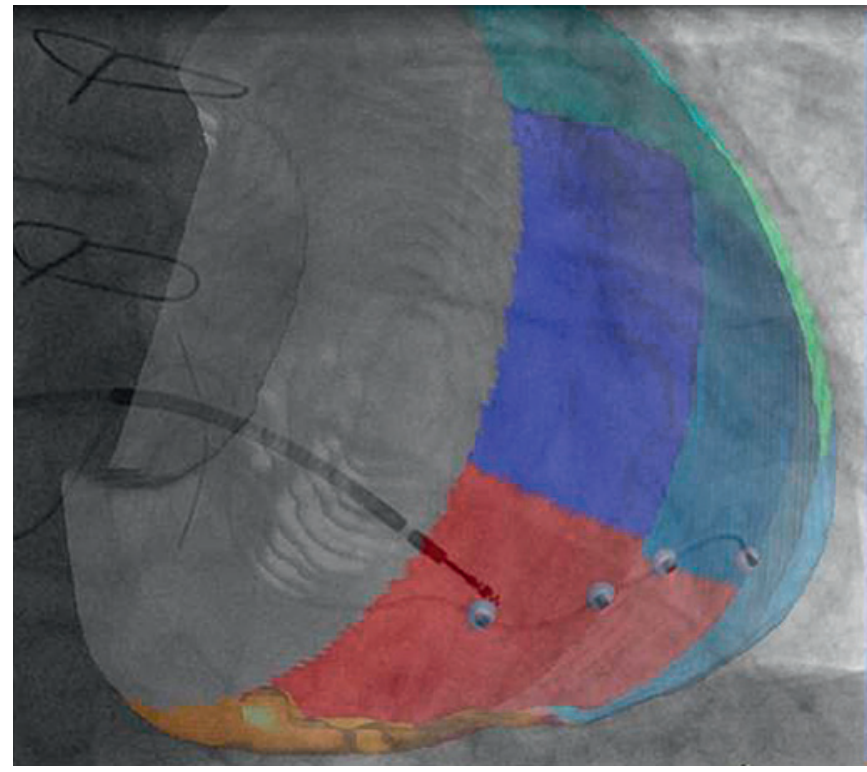

Abstract 152 Figure 2

\section{VENTRICULAR PACING ALONG INDIVIDUAL BRANCHES OF THE CORONARY SINUS USING A OUADRIPOLAR LV PACING LEAD}

doi:10.1136/heartjnl-2011-300198.153

${ }^{1,2} \mathrm{~A}$ K Shetty, 1,2 P Mehta, 1,2S Duckett, ${ }^{1,2} \mathrm{~J}$ Bostock, ${ }^{1,2} \mathrm{M}$ Ginks, ${ }^{1,2} \mathrm{~S}$ Hamid, ${ }^{1,2} \mathrm{M}$ Sohal, ${ }^{1,2} \mathrm{R}$ Razavi, ${ }^{1,2} \mathrm{Y} \mathrm{Ma},{ }^{1,2} \mathrm{~K}$ Rhode, ${ }^{1,2} \mathrm{~A}$ Arujuna, ${ }^{1,2} \mathrm{C}$ A Rinaldi. ${ }^{1}$ Guys and $\mathrm{St}$ Thomas' Hospital NHS Foundation Trust; ${ }^{2}$ King's College London, London, UK

Introduction Cardiac resynchronisation therapy (CRT) usually involves placing the left ventricular (LV) pacing lead in the posterolateral or lateral region of the LV epicardial surface as this is thought likely to re-coordinate myocardial contraction most effectively. The LV lead is standardly placed in a position with the best pacing parameters and satisfactory stability. It is not known, however, whether there is a significant difference in haemodynamic response to LV pacing in different regions of the same coronary sinus (CS) vein. In this study we aimed to evaluate the difference in acute haemodynamic response to pacing along individual branches of the CS.

Methods 16 patients underwent an acute haemodynamic study during their CRT-defibrillator implant. We used a high fidelity pressure wire to assess the acute haemodynamic response (AHR) to pacing in different branches of the coronary sinus. We used a novel quadripolar lead (Quartet, St Jude Medical, Sylmar, California, USA) that has four poles on the LV lead-distal tip and 3 ring electrodes. The 3 ring electrodes are spaced $20 \mathrm{~mm}, 30 \mathrm{~mm}$ and $47 \mathrm{~mm}$ from the distal tip electrode and the four poles allow bipolar pacing between them. It was thus possible for us to test pacing parameters and AHR along a significant proportion of a CS branch without having to reposition the LV lead.

Results DDDLV pacing was attempted in as many different CS branches as possible in each patient (total 56 different positions used). The mean overall percentage difference in AHR (measured by change in $+\mathrm{dP} / \mathrm{dt}$ compared to baseline AAI pacing or VVI pacing in AF patients) between an individual CS branch bipole with the lowest $+\mathrm{dP} / \mathrm{dt}$ and that with the highest was $6.6 \pm 5.6 \%$. Much larger differences in change in $+\mathrm{dP} / \mathrm{dt}$ were seen, however, between different branches of the CS in the same patient with a mean difference in change in $+\mathrm{dP} / \mathrm{dt}$ in the best CS vein compared to the worst CS vein of $16.7 \pm 6.3 \%$. Although the difference in AHR seen between different bipoles within the same vein were not large, we did find that in some cases no pacing capture was found with one 
bipole but was found with another. Furthermore, differences in whether phrenic nerve stimulation (PNS) occurred were seen when using different LV lead bipoles within the same branch of the CS. Conclusion Our data suggest that only a small difference in AHR is seen when pacing along the same branch of the CS compared to pacing within different branches of the CS within the same patient. This means that although the site of LV lead placement is important, a proximal or distal position within a CS branch is much less important than choosing the right branch in terms of acute haemodynamic response. A choice of bipoles on the LV lead may mean, however, that problems with capture thresholds or PNS can be overcome without the need to reposition the LV lead.

\section{4}

\section{PATIENTS RECEIVING STANDARD PACEMAKER GENERATOR REPLACEMENTS FREQUENTLY HAVE IMPAIRED LEFT VENTRICULAR FUNCTION AND EXERCISE INTOLERANCE, RELATED TO THE PERCENTAGE OF RIGHT VENTRICULAR PACING}

doi:10.1136/heartjnl-2011-300198.154

${ }^{1} \mathrm{G}$ A Begg, ${ }^{1} \mathrm{~J}$ Gierula, ${ }^{1} \mathrm{Z} \mathrm{L}$ Waldron, ${ }^{2} \mathrm{~K}$ K Witte. ' Leeds General Infirmary, Leeds, UK; ${ }^{2}$ University of Leeds, Leeds, UK

Background Right ventricular (RV) pacing is an accepted treatment for symptomatic bradycardia. However, long-term RV pacing is increasingly recognised to be detrimental to left ventricular (LV) systolic function. We wanted to establish the prevalence, associated features and predictors of LV systolic dysfunction (LVSD) and outcome in a contemporary group of patients with long-term RV pacemakers.

Methods We prospectively recruited consecutive patients listed for PGR between 2008 and 2010 at Leeds General Infirmary. We performed echocardiography, exercise testing and recorded indications for pacing, pacing variables and duration of pacing, comorbidities, current medication and renal function.

Results Of 399 PGR procedures 342 subjects (86\%), 184 men, attended. Non-attendees had similar pacing variables and were of similar age as attendees. Mean age (SE) was 76 (1), and mean duration of pacing was 10 (0.3) years. Comorbidites were common: diabetes mellitus in $11 \%$, previous myocardial infarction in $15 \%$, previous cardiac surgery in $26 \%$ and atrial fibrillation (AF) in $26 \%$. Medical therapy included $\beta$-blockers in $60 \%$ and ACE inhibitors in $70 \%$. Dual chamber devices were implanted in $77 \%$ (45\% of all patients had rate responsive (RR) pacing programmed). Mean percentage of ventricular pacing (\%VP) was $61(2) \%$. Mean left ventricular ejection fraction (LVEF) was 49 (1)\%, (44\% had an LVEF $<50 \%$ ). Mean peak oxygen uptake $\left(\mathrm{pVo}_{2}\right.$ ) (in 107 subjects) was 17 (1) $\mathrm{ml} / \mathrm{kg} / \mathrm{min}$ and mean creatinine was 108 (3) $\mu \mathrm{mol} / \mathrm{l}$. There was an inverse relationship between LVEF and \%VP $(0.42 ; p<0.0001)$, and years since first implanted $(p=0.09)$ but there was no effect on LVEF of age, the presence of AF and the pacing mode. In single chamber devices, RR pacing was associated with higher \%VP $(p=0.01)$, and a trend to worse LVEF $(p=0.09)$. These differences were not seen in RR programmed dual chamber devices. There was a negative relationship between $\mathrm{pVo}_{2}$ and $\% \mathrm{VP}(\mathrm{r}=0.21 ; \mathrm{p}<0.03)$. Even with a short follow-up period of $16(0.5)$ months, 23 (7\%) patients are dead. Patients dead at the censor date were older at the time of the assessment $(p<0.005)$, had a higher \%VP $(p<0.03)$ and worse renal function $(p<0.001)$, but did not have significantly worse LVEF or $\mathrm{pVO}_{2}$. The presence of a single chamber device was associated with a poorer outcome $(\mathrm{p}<0.002)$ despite patients with a single chamber device being of similar age as those with a dual chamber device.

Conclusions Patients receiving standard pacemaker generator replacements frequently have cardiovascular comorbidities, left ventricular dysfunction and impaired $\mathrm{pVO}_{2}$ and suffer a high mortality rate. In an unselected population of patients with pacemakers, we have established that the amount of RV pacing is related not only to important surrogate measures of outcome such as exercise tolerance and LVEF but also mortality. Whether an aggressive policy of limiting RV pacing in patients at risk reduces mortality is unknown.

\section{INCIDENCE SCREENING OF PATIENTS FOLLOWING ST ELEVATION MYOCARDIAL INFARCTION FOR PRIMARY PREVENTION IMPLANTABLE CARDIOVERTER DEFIBRILLATOR (ICD) IMPLANTATION HAS A LOW THERAPEUTIC YIELD}

doi:10.1136/heartjnl-2011-300198.155

E L Berry, H C Padgett, A J Ahsan, A D Staniforth. Nottingham University Hospitals NHS Trust, Nottingham, UK

Introduction The ICD implant rate for the United Kingdom is low compared with the European Union and United States of America. National Institute of Clinical Excellence guidance TAO95 (NICE 2006) makes recommendations for primary prevention ICD implantation. Our study investigated the feasibility of systematically screening patients following an acute ST elevation myocardial infarction (STEMI) to improve local ICD implant rates.

Method A prospective single centre study was performed over 14months, in tertiary centre setting. All patients with a diagnosis of an acute STEMI had an echocardiogram at 6 weeks to assess left ventricular ejection fraction (LVEF). Patients with impaired LVEF then underwent screening for primary prevention ICD as per TA095 recommendations (Abstract 155 figure 1).

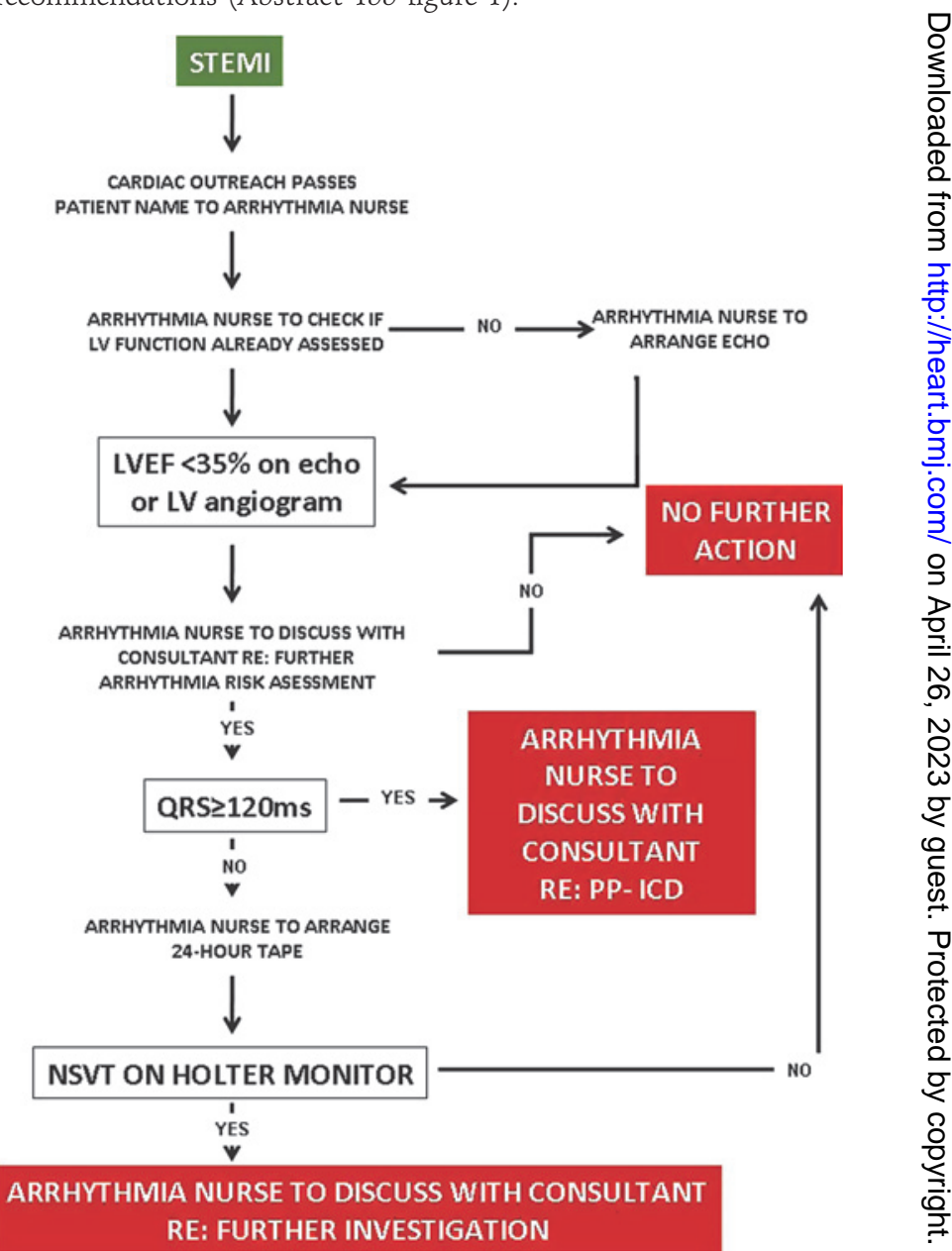

Abstract 155 Figure 1 